T. E. DUNCA N (Lawrence, KS)

B. P A S IK-DUNCA N (Lawrence, KS)

E. STETTNER (Warszawa)

\title{
ADAPTIVE CONTROL OF DISCRETE TIME MARKOV PROCESSES BY THE LARGE DEVIATIONS METHOD
}

Abstract. Some discrete time controlled Markov processes in a locally compact metric space whose transition operators depend on an unknown parameter are described. The adaptive controls are constructed using the large deviations of empirical distributions which are uniform in the parameter that takes values in a compact set. The adaptive procedure uses a finite family of continuous, almost optimal controls. Using the large deviations property it is shown that an adaptive control which is a fixed almost optimal control after a finite time is almost optimal with probability nearly 1 .

0. Introduction. Consider a controlled Markov process $\left(x_{n}, n \in \mathbb{N}\right)$ on a probability space $(\Omega, F, P)$ taking values in a locally compact metric space $\left(E, \varrho_{E}\right)$ with the transition operator $P^{\alpha^{0}, v_{n}}\left(x_{n}, \cdot\right)$ at time $n$. The quantity $\alpha^{0}$ is an unknown parameter that is an element of a compact metric space $\left(\mathbb{A}, \varrho_{\mathbb{A}}\right)$, and the term $v_{n}$ that is the control is a $\sigma\left(x_{0}, \ldots, x_{n}\right)$-adapted random variable with values in a compact metric space $\left(U, \varrho_{U}\right)$.

Let $c: E \times U \rightarrow \mathbb{R}_{+}$be a continuous bounded function and let

$$
J^{\alpha^{0}}\left(\left(v_{n}, n \in \mathbb{N}\right)\right)=\limsup _{n \rightarrow \infty} \frac{1}{n} \sum_{i=0}^{n-1} c\left(x_{i}, v_{i}\right) .
$$

The control problem is to minimize $J$ over the admissible strategies $\left(v_{n}, n \in \mathbb{N}\right)$ where $v_{n}$ is a $U$-valued, $\sigma\left(x_{0}, \ldots, x_{n}\right)$-adapted random vari-

2000 Mathematics Subject Classification: 93E20, 93E10, 93C40.

Key words and phrases: adaptive control, discrete time controlled Markov processes, large deviations.

Research supported in part by NSF Grant DMS 9623439 and KBN Grant 2 P03A 05309. 
able. If $\alpha^{0}$ is known and some ergodic assumptions are satisfied, then the family of admissible strategies can be restricted to Markovian ones, that is, $v_{n}=u\left(x_{n}\right)$, where $u \in \mathcal{A}=B(E, U)$, the family of Borel measurable functions from $E$ to $U$. Thus $J^{\alpha^{0}}\left(\left(u\left(x_{n}\right), n \in \mathbb{N}\right)\right)$ is minimized over $u \in \mathcal{A}$.

If $\alpha^{0}$ is unknown then an estimate of $\alpha^{0}$ is made for each $n \in \mathbb{N}$ using the state $x_{n}$ and a control $u \in \mathcal{A}$ is chosen that is almost optimal for the current value of the estimate of $\alpha^{0}$. While such a strategy can be shown to be almost self-optimizing (cf., e.g., $[5,9]$ ) the procedure requires an estimate for each $n \in \mathbb{N}$ and a choice of a control. In the approach in this paper an adaptive control is fixed after a finite time and it is shown to be almost optimal with probability nearly 1 . This approach uses the results on the large deviations of empirical distributions which are uniform in the parameter. These large deviation results are described in Section 2. In Section 3 it is shown that an adaptive control can be taken to be fixed after a finite time and almost optimal. In Section 4 two Markov models are given for which the results in Sections 2 and 3 can be applied.

Throughout the paper we denote by:

- $\mathcal{C}(E)$ the space of continuous bounded functions on $E$,

- $\mathcal{P}(E)$ the space of all probability measures on $E$ endowed with the weak convergence topology and with the set of Borel subsets $\mathcal{B}(\mathcal{P}(E))$,

- $\mathcal{B}(E)$ the set of Borel subsets of $E$.

2. Uniform large deviations of empirical distributions. In this section we consider an uncontrolled Markov process $\left(x_{n}, n \in \mathbb{N}\right)$ with transition operators $P^{\alpha}\left(x_{n}, \cdot\right)$ where $\alpha \in \mathbb{A}$. The following assumptions are made:

For $f \in \mathcal{C}(E)$ the mapping

$$
A \times E \ni(\alpha, x) \mapsto P^{\alpha} f(x):=\int_{E} f(y) P^{\alpha}(x, d y)
$$

is continuous.

For $B \in \mathcal{B}(E)$ and $C \in \mathcal{B}(\mathcal{P}(E))$, the empirical measure and its probability (see [2] and [3]) are defined as follows:

$$
\begin{aligned}
S_{n}(B) & =\frac{1}{n} \sum_{i=0}^{n-1} \chi_{B}\left(x_{i}\right), \\
Q_{n x}^{\alpha}(C) & =P_{x}^{\alpha}\left\{S_{n} \in C\right\},
\end{aligned}
$$

where $P_{x}^{\alpha}$ stands for the conditional probability measure given $\left(x_{n}, n \in \mathbb{N}\right)$ starts from $x$ and the true parameter is $\alpha$. Furthermore let

$$
\Phi:=\{f \in \mathcal{C}(E): \exists a>0 \forall x \in E f(x) \geq a\}
$$


and for $\mu \in \mathcal{P}(E)$, define

$$
I^{\alpha}(\mu)=\sup _{f \in \Phi} \int_{E} \log \frac{f(x)}{P^{\alpha} f(x)} \mu(d x) .
$$

THEOREM 1. If (B1) is satisfied then for compact subsets $C$ and $A_{1}$ of $\mathcal{P}(E)$ and $\mathbb{A}$, respectively, we have

$$
\limsup _{n \rightarrow \infty} n^{-1} \sup _{\alpha \in A_{1}} \sup _{x \in E} \log Q_{n x}^{\alpha}(C) \leq-\inf _{\alpha \in A_{1}} \inf _{\mu \in C} I^{\alpha}(\mu)
$$

where $Q$ is given by (3) and $I$ is given by (5).

Proof. For $d>1$ let

$$
\Phi_{d}=\left\{f \in \Phi: \sup _{x \in E} f(x) \leq d \inf _{x \in E} f(x)\right\}
$$

and for $\mu \in \mathcal{P}(E)$ let

$$
I_{d}^{\alpha}(\mu)=\sup _{f \in \Phi_{d}} \int_{E} \log \frac{f(x)}{P^{\alpha} f(x)} \mu(d x) .
$$

Clearly

$$
\begin{aligned}
E_{x}^{\alpha}\left\{\exp \left\{\sum_{i=0}^{n-1} \log \left(\frac{f\left(x_{i}\right)}{P^{\alpha} f\left(x_{i}\right)}\right)\right\}\right\} & E_{x}^{\alpha}\left\{\exp \left\{n \int_{E} \log \frac{f(y)}{P^{\alpha} f(y)} S_{n}(d y)\right\}\right\} \\
& =\int_{\mathcal{P}(E)} \exp \left\{n \int_{E} \log \frac{f(y)}{P^{\alpha} f(y)} \mu(d y)\right\} Q_{n x}^{\alpha}(d \mu) .
\end{aligned}
$$

Since by the definition of the family $\Phi_{d}$, for $n=1,2, \ldots$,

$$
E_{x}^{\alpha}\left\{\exp \left\{\sum_{i=0}^{n-1} \log \frac{f\left(x_{i}\right)}{P^{\alpha} f\left(x_{i}\right)}\right\}\right\} \leq d
$$

by (7) it follows that

$$
\int_{\mathcal{P}(E)} \exp \left\{n \int_{E} \log \frac{f(y)}{P^{\alpha} f(y)} \mu(d y)\right\} Q_{n x}^{\alpha}(d \mu) \leq d .
$$

Consequently, for any Borel subset $\Gamma$ of $\mathcal{P}(E)$ and $\alpha \in \mathbb{A}$,

$$
\sup _{x \in E} Q_{n x}^{\alpha}(\Gamma) \leq d \exp \left(-n \inf _{\mu \in \Gamma} \int_{E} \log \frac{f(y)}{P^{\alpha} f(y)} \mu(d y)\right) .
$$

Now let

$$
\kappa_{d}=\inf _{\alpha \in A_{1}} \inf _{\mu \in C} I_{d}^{\alpha}(\mu)
$$


Since each $f \in \Phi$ belongs to $\Phi_{d}$ with $d$ sufficiently large, for $f \in \Phi$ the mapping

$$
\mathcal{P}(E) \times \mathbb{A} \ni(\mu, \alpha) \mapsto \mu\left(\log \frac{f}{P^{\alpha} f}\right):=\int_{E} \log \frac{f(y)}{P^{\alpha} f(y)} \mu(d y)
$$

is continuous. Therefore, for $\varepsilon>0$ the set

is open and

$$
\left\{(\mu, \alpha) \in \mathcal{P}(E) \times \mathbb{A}: \mu\left(\log \frac{f}{P^{\alpha} f}\right)>\kappa_{d}-\varepsilon\right\}
$$

$$
C \times A_{1} \subset \bigcup_{f \in \Phi_{d}}\left\{(\mu, \alpha): \mu\left(\log \frac{f}{P^{\alpha} f}\right)>\kappa_{d}-\varepsilon\right\} .
$$

Since $C \times A_{1}$ is a compact subset of $\mathcal{P}(E) \times \mathbb{A}$, there is a finite subset $\left\{f_{1}, \ldots, f_{k}\right\}$ of $\Phi_{d}$ such that

$$
C \times A_{1} \subset \bigcup_{j=1}^{k}\left\{(\mu, \alpha): \mu\left(\log \frac{f_{j}}{P^{\alpha} f_{j}}\right)>\kappa_{d}-\varepsilon\right\} .
$$

Consequently, for every $\alpha \in A_{1}$,

$$
C \subset \bigcup_{f \in \Phi_{d}}\left\{\mu \in \mathcal{P}(E): \mu\left(\log \frac{f}{P^{\alpha} f}\right)>\kappa_{d}-\varepsilon\right\} .
$$

Now if we replace $\Gamma$ in (10) by the sets

$$
K_{j}^{\alpha}=\left\{\mu \in \mathcal{P}(E): \mu\left(\log \frac{f_{j}}{P^{\alpha} f_{j}}\right)>\kappa_{d}-\varepsilon\right\} \cap C
$$

it follows that

$$
\sup _{x \in E} Q_{n x}^{\alpha}\left(K_{j}^{\alpha}\right) \leq d e^{-n\left(\kappa_{d}-\varepsilon\right)}
$$

Hence

$$
\sup _{x \in E} \sup _{\alpha \in A_{1}} Q_{n x}^{\alpha}(C) \leq \sup _{x \in E} \sup _{\alpha \in A_{1}}\left[\sum_{j=1}^{k} Q_{n x}^{\alpha}\left(K_{j}^{\alpha}\right)\right] \leq k d e^{-n\left(\kappa_{d}-\varepsilon\right)}
$$

and

$$
\limsup _{n \rightarrow \infty} \sup _{x \in E} \sup _{\alpha \in A_{1}} n^{-1} \log Q_{n x}^{\alpha}(C) \leq-\kappa_{d}+\varepsilon .
$$

Since $\varepsilon>0$ can be chosen arbitrarily small, it follows that

$$
\limsup _{n \rightarrow \infty} \sup _{x \in E} \sup _{\alpha \in A_{1}} n^{-1} \log Q_{n x}^{\alpha}(C) \leq-\kappa_{d} .
$$

To complete the proof it remains to show that

$$
\lim _{d \rightarrow \infty} \kappa_{d}=\inf _{\alpha \in A_{1}} \inf _{\mu \in C} I^{\alpha}(\mu) .
$$


Note that for $\varepsilon>0$,

$$
C \times A_{1} \subset \bigcup_{f \in \Phi}\left\{(\mu, \alpha): \mu\left(\log \frac{f}{P^{\alpha} f}\right)>\inf _{\alpha \in A_{1}} \inf _{\mu \in C} I^{\alpha}(\mu)-\varepsilon\right\}
$$

and by the compactness of $C \times A_{1}$ there is a finite set $\left\{f_{1}, \ldots, f_{k}\right\} \subset \Phi$ such that

$$
C \times A_{1} \subset \bigcup_{j=1}^{k}\left\{(\mu, \alpha): \mu\left(\log \frac{f_{j}}{P^{\alpha} f_{j}}\right)>\inf _{\alpha \in A_{1}} \inf _{\mu \in C} I^{\alpha}(\mu)-\varepsilon\right\} .
$$

By the definitions of $\Phi$ and $\Phi_{d}$ there is $d>0$ such that $\left\{f_{1}, \ldots, f_{k}\right\} \subset \Phi_{d}$. Therefore, for each $\alpha \in A_{1}$ and $\mu \in C$,

$$
\begin{aligned}
\sup _{f \in \Phi_{d}} \int_{E} \log \frac{f(y)}{P^{\alpha} f(y)} \mu(d y) & \geq \max _{i=1, \ldots, k} \int_{E} \log \frac{f_{i}(y)}{P^{\alpha} f_{i}(y)} \mu(d y) \\
& >\inf _{\alpha \in A_{1}} \inf _{\mu \in C} I^{\alpha}(\mu)-\varepsilon
\end{aligned}
$$

and

$$
\limsup _{d \rightarrow \infty} k_{d} \geq \inf _{\alpha \in A_{1}} \inf _{\mu \in C} I^{\alpha}(\mu)-\varepsilon
$$

Since the last inequality holds for any $\varepsilon>0$, and

$$
\kappa_{d} \leq \inf _{\alpha \in A_{1}} \inf _{\mu \in C} I^{\alpha}(\mu),
$$

the equality (14) follows, which completes the proof.

In the proof of Theorem 1 the compactness of the set $C \subset \mathcal{P}(E)$ is important. To relax this requirement an extra assumption on the transition operator $P^{\alpha}(x, \cdot)$ is made:

(B2) There is a continuous function $\psi: \mathbb{A} \times E \rightarrow \mathbb{R}$ such that $\psi(\alpha, x) \geq 1$ for $\alpha \in \mathbb{A}, x \in E$, the mapping

$$
E \ni x \mapsto \sup _{\alpha \in A} \int_{E} \psi(\alpha, y) P^{\alpha}(x, d y)
$$

is bounded on compact subsets of $E$ and for each $m>0$ the set

$$
K_{m}:=\left\{x \in E: \inf _{\alpha \in A} \frac{\psi(\alpha, x)}{\int_{E} \psi(\alpha, y) P^{\alpha}(x, d y)} \leq m\right\}
$$

is compact.

Let

$$
\varrho:=\inf _{x \in E} \inf _{\alpha \in \mathbb{A}} \frac{\psi(\alpha, x)}{\int_{E} \psi(\alpha, y) P^{\alpha}(x, d y)} .
$$

If (B2) is satisfied then $\varrho>0$. The following two lemmas are easy adaptations of Lemmas 4.1 and 4.3 of [3]. 
LEMma 1. If (B2) is satisfied then for each $\varepsilon>0$,

$$
\begin{aligned}
Q_{n x}^{\alpha}\left(\left\{\mu \in \mathcal{P}(E): \mu\left(K_{m}^{\mathrm{c}}\right)>\right.\right. & \varepsilon\}) \\
& \leq \psi(\alpha, x) \exp \left\{-n \log \varrho-n \varepsilon \log \frac{m}{\varrho}\right\} .
\end{aligned}
$$

LEMMA 2. If (B2) is satisfied then for any real number a and any compact set $W \subset E$ there exists a compact subset $C(a) \subset \mathcal{P}(E)$ such that

$$
\limsup _{n \rightarrow \infty} n^{-1} \sup _{\alpha \in \mathbb{A}} \sup _{x \in W} Q_{n x}^{\alpha}\left(C(a)^{(\mathrm{c})}\right) \leq-a .
$$

Using Lemma 2, one can generalize Theorem 1 in the same way as in Theorem 4.4 of [3].

THEOREM 2. If (B1) and (B2) are satisfied, then for any closed subsets $C$ and $A_{1}$ of $\mathcal{P}(E)$ and $\mathbb{A}$, respectively, and compact subsets $W$ of $E$,

$$
\limsup _{n \rightarrow \infty} n^{-1} \sup _{\alpha \in A_{1}} \sup _{x \in W} \log Q_{n x}^{\alpha}(C) \leq-\inf _{\alpha \in A_{1}} \inf _{\mu \in C} I^{\alpha}(\mu) .
$$

Subsequently, the following lemma is used.

Lemma 3. If (B1) and (B2) are satisfied then for each $m>0$ the set

$$
C_{m}=\left\{\mu \in \mathcal{P}(E): \inf _{\alpha \in A} I^{\alpha}(\mu) \leq m\right\}
$$

is compact in $\mathcal{P}(E)$.

Proof. Since for each fixed $\mu \in C_{m}$ the mapping $\alpha \mapsto I^{\alpha}(\mu)$ is lower semicontinuous, there is an $\alpha(\mu) \in \mathbb{A}$ such that $I^{\alpha}(\mu) \leq m$. By (B2) for $r>0$ the set

$$
K_{r}^{\alpha}=\left\{x \in E: \frac{\psi(\alpha, x)}{\int_{E} \psi(\alpha, y) P^{\alpha}(x, d y)} \leq r\right\} \subset K_{r}
$$

is compact in $E$ where $K_{r}$ is defined in (16). Adapting the method of Lemma 4.2 of [3] it can be shown that for $r>\varrho$,

$$
\mu\left(\left(K_{r}^{\alpha}\right)^{\mathrm{c}}\right) \leq \frac{m-\log \varrho}{\log r-\log \varrho}<\varepsilon
$$

Therefore, for $\varepsilon>0$ there is $r>0$ such that for any $\mu \in C_{m}$,

$$
\mu\left(\left(K_{r}\right)^{\mathrm{c}}\right) \leq \mu\left(\left(K_{r}^{\alpha(\mu)}\right)^{\mathrm{c}}\right) \leq \varepsilon .
$$

Consequently, the family $C_{m}$ of measures is tight. Since the mapping

$$
\mathbb{A} \times \mathcal{P}(E) \ni(\alpha, \mu) \mapsto I^{\alpha}(\mu)
$$

is lower semicontinuous, the set $C_{m}$ is compact.

In the next proposition the rate functional $I^{\alpha}(\mu)$ is shown to be positive.

Proposition 1. Assume (B1), (B2) and that for each $\alpha \in \mathbb{A}$ there is a unique invariant measure $\pi_{\alpha}$ of the transition operator $P^{\alpha}(x, \cdot)$. Let $A_{1}$ and 
$C$ be closed subsets of $\mathbb{A}$ and $\mathcal{P}(E)$ such that $\pi_{\alpha} \notin C$ for $\alpha \in A_{1}$. Then

$$
\inf _{\alpha \in A_{1}} \inf _{\mu \in C} I^{\alpha}(\mu)>0 .
$$

P r o of. Note first that the mapping (15) is lower semicontinuous and therefore it attains its minimum on compact sets. Moreover $I^{\alpha}(\mu) \geq 0$ for $\mu \in \mathcal{P}(E)$ and $\alpha \in \mathbb{A}$ and, by Lemma 2.5 of $[3], I^{\alpha}(\mu)=0$ if and only if $\mu=\pi_{\alpha}$.

By the compactness of the set $C_{m}$, defined in (21), for each $m>0$ it follows that

$$
\inf _{\alpha \in A_{1}} \inf _{\mu \in C \cap C_{m}} I^{\alpha}(\mu)>0 .
$$

Since by the definition (21),

$$
\inf _{\alpha \in \mathbb{A}} \inf _{\mu \in C_{m}^{c}} I^{\alpha}(\mu) \geq m>0
$$

(23) is verified.

An additional assumption is made.

(B3) For each $\alpha$ there is a unique invariant measure $\pi_{\alpha}$ for the transition operator $P^{\alpha}(x, \cdot)$ and for $c \in \mathcal{C}(E)$ the mapping $\mathbb{A} \ni \alpha \mapsto \pi_{\alpha}(c)$ is continuous.

Using the assumptions (B1)-(B3) we give a uniform estimate of the deviations of the running costs from the limit.

THEOREM 3. If (B1)-(B3) are satisfied, then for $\varepsilon>0, c \in \mathcal{C}(E)$ and any compact set $W \subset E$ there are a $p>0$ and a positive integer $N$ such that for $n \geq N$,

$$
\sup _{\alpha \in A} \sup _{x \in W} P_{x}^{\alpha}\left\{\left|n^{-1} \sum_{j=0}^{n-1} c\left(x_{j}\right)-\pi_{\alpha}(c)\right| \geq \varepsilon\right\} \leq e^{-n p} .
$$

Proof. By (B3) for $\varepsilon>0$ and $\alpha \in \mathbb{A}$ there exists $\delta_{\alpha}>0$ such that if $\alpha^{\prime} \in B\left(\alpha, \delta_{\alpha}\right):=\left\{\bar{\alpha} \in \mathbb{A}: \varrho_{A}(\alpha, \bar{\alpha}) \leq \delta_{\alpha}\right\}$, then $\left|\pi_{\alpha}(c)-\pi_{\alpha^{\prime}}(c)\right| \leq \varepsilon$. Since $\mathbb{A}$ is compact there is a finite set $\alpha_{1}, \ldots, \alpha_{k}$ such that $\mathbb{A} \subset \bigcup_{i=1}^{k} B\left(\alpha_{i}, \delta_{\alpha_{i}}\right)$. For $i=1, \ldots, k$ let $C_{\varepsilon}\left(\alpha_{i}\right):=\left\{\mu \in \mathcal{P}(E):\left|\mu(c)-\pi_{\alpha_{i}}(c)\right| \geq 2 \varepsilon\right\}$. Clearly $C_{\varepsilon}\left(\alpha_{i}\right)$ is a closed subset of $\mathcal{P}(E)$ and if $\alpha \in B\left(\alpha_{i}, \delta_{\alpha_{i}}\right)$ then $\pi_{\alpha} \notin C_{\varepsilon}\left(\alpha_{i}\right)$. Therefore, by Proposition 1 and Theorem 2 there are $p>0$ and $N>0$ such that for $n>N$ and $i=1, \ldots, k$,

$$
\sup _{\alpha \in B\left(\alpha_{i}, \delta_{\alpha_{i}}\right)} \sup _{x \in W} Q_{n x}^{\alpha}\left(C_{\varepsilon}\left(\alpha_{i}\right)\right) \leq e^{-n p} .
$$

Equivalently this means that

$$
\sup _{\alpha \in B\left(\alpha_{i}, \delta_{\alpha_{i}}\right)} \sup _{x \in W} P_{x}^{\alpha}\left\{\left|n^{-1} \sum_{j=0}^{n-1} c\left(x_{j}\right)-\pi_{\alpha_{i}}(c)\right| \geq 2 \varepsilon\right\} \leq e^{-n p}
$$


for $i=1, \ldots, k$. By the definition of $B\left(\alpha_{i}, \delta_{\alpha_{i}}\right)$ it follows that

$$
\sup _{\alpha \in B\left(\alpha_{i}, \delta_{\alpha_{i}}\right)} \sup _{x \in W} P_{x}^{\alpha}\left\{\left|n^{-1} \sum_{j=0}^{n-1} c\left(x_{j}\right)-\pi_{\alpha}(c)\right| \geq \varepsilon\right\} \leq e^{-n p}
$$

for $i=1, \ldots, k$ and consequently (24) is obtained.

The remaining part of this section is devoted to the study of the large deviations for empirical distributions of pairs of consecutive states. By analogy to (2) and (3), for $B_{1}, B_{2} \in \mathcal{B}(E)$ let

$$
\bar{S}_{n}\left(B_{1} \times B_{2}\right)=\frac{1}{n} \sum_{i=0}^{n-1} \chi_{B_{1}}\left(x_{i}\right) \chi_{B_{2}}\left(x_{i+1}\right)
$$

and for $C \in \mathcal{B}(\mathcal{P}(E \times E))$ let

$$
\bar{Q}_{n x}^{\alpha}(C)=P_{x}^{\alpha}\left\{\bar{S}_{n} \in C\right\} .
$$

By analogy to (4) and (5) define

$$
\bar{\Phi}:=\{f \in C(E \times E): \exists a>0 \forall x, y \in E f(x, y) \geq a\}
$$

and for $\mu \in \mathcal{P}(E \times E)$,

$$
\bar{I}^{\alpha}(\mu)=\sup _{f \in \bar{\Phi}} \int_{E} \log \frac{f(x, y)}{P^{\alpha} f(y)} \mu(d x, d y)
$$

with $P^{\alpha} f(y)=\int_{E} f(y, z) P^{\alpha}(y, d z)$.

THEOREM 4. If (B1) holds then for any compact subsets $C \subset \mathcal{P}(E \times E)$ and $A_{1} \subset \mathbb{A}$,

$$
\limsup _{n \rightarrow \infty} n^{-1} \sup _{\alpha \in A_{1}} \sup _{x \in E} \log \bar{Q}_{n x}^{\alpha}(C) \leq-\inf _{\alpha \in A_{1}} \inf _{\mu \in C} \bar{I}^{\alpha}(\mu) .
$$

Proof. Note first that if $f \in \bar{\Phi}_{d}$, where

$$
\bar{\Phi}_{d}:=\left\{f \in C(E \times E): \sup _{x, y \in E} f(x, y) \leq d \inf _{x, y \in E} f(x, y)\right\},
$$

then

$$
E_{x}^{\alpha}\left\{\exp \left\{\sum_{i=0}^{n-1} \log \frac{f\left(x_{i}, x_{i+1}\right)}{P^{\alpha} f\left(x_{i+1}\right)}\right\}\right\} \leq d .
$$

If $\Gamma \in \mathcal{B}(\mathcal{P}(E \times E))$ and $\alpha \in \mathbb{A}$ then

$$
\sup _{x \in E} \bar{Q}_{n x}^{\alpha}(\Gamma) \leq d \exp \left(-n \inf _{\mu \in \Gamma} \int_{E} \log \frac{f(x, y)}{P^{\alpha} f(y)} \mu(d x, d y)\right)
$$

and the proof of Theorem 1 can be easily modified. 
REMARK 1. If $\bar{I}^{\alpha}$ is defined as

$$
\sup _{f \in \bar{\Phi}} \int_{E} \log \frac{f(x, y)}{P^{\alpha} f(x)} \mu(d x, d y)
$$

then following Section 1.3 of [7] an analogue of Theorem 4 can be obtained with a simpler proof because

$$
E_{x}^{\alpha}\left\{\exp \left\{\sum_{i=0}^{n-1} \log \frac{f\left(x_{i}, x_{i+1}\right)}{P^{\alpha} f\left(x_{i}\right)}\right\}\right\}=1 .
$$

However to adapt Theorem 4 to the case of a noncompact closed set $C$ the rate functional $\bar{I}^{\alpha}$ of the form defined in (28) is required.

An additional assumption is made now.

(B4) There is a continuous function $\bar{\psi}: \mathbb{A} \times E \times E \rightarrow \mathbb{R}$ such that $\bar{\psi}(\alpha, x, y) \geq 1$ for $\alpha \in \mathbb{A}$ and $x, y \in E$, the mapping

$$
E \ni x \mapsto \sup _{\alpha \in \mathbb{A}} \int_{E} \bar{\psi}(\alpha, x, y) P^{\alpha}(x, d y)
$$

is bounded on compact sets and for each $m>0$ the set

$$
\bar{K}_{m}:=\left\{(x, y) \in E \times E: \inf _{\alpha \in \mathbb{A}} \frac{\bar{\psi}(\alpha, x, y)}{\int_{E} \bar{\psi}(\alpha, x, y) P^{\alpha}(y, d z)} \leq m\right\}
$$

is compact in $E \times E$.

Let

$$
\bar{\varrho}:=\inf _{x, y \in E} \inf _{\alpha \in \mathbb{A}} \frac{\bar{\psi}(\alpha, x, y)}{\int_{E} \bar{\psi}(\alpha, x, y) P^{\alpha}(y, d z)} .
$$

Adapting Lemmas 1-3 and Theorem 2 to the case of consecutive pairs easily yields the following results.

Lemma 4. If (B4) is satisfied, then for each $\varepsilon>0$,

$$
\begin{aligned}
Q_{n x}^{\alpha}\left(\left\{\mu \in \mathcal{P}(E \times E): \mu\left(\bar{K}_{m}^{c}\right)>\varepsilon\right\}\right) & \\
& \leq \bar{\psi}(\alpha, x, y) \exp \left\{-n \log \bar{\varrho}-n \varepsilon \log \frac{m}{\bar{\varrho}}\right\} .
\end{aligned}
$$

LEMMA 5. If (B4) is satisfied, then for any real number $a$ and any compact set $W \subset E$ there exists a compact subset $C(a) \subset \mathcal{P}(E \times E)$ such that

$$
\limsup _{n \rightarrow \infty} n^{-1} \sup _{\alpha \in \mathbb{A}} \sup _{x \in W} \bar{Q}_{n x}^{\alpha}\left(C(a)^{\mathrm{c}}\right) \leq-a .
$$


THEOREM 5. If (B1) and (B4) are satisfied, then for any closed subsets $C$ of $\mathcal{P}(E \times E)$ and $A_{1}$ of $\mathbb{A}$, and any compact subset $W$ of $E$,

$$
\limsup _{n \rightarrow \infty} n^{-1} \sup _{\alpha \in A_{1}} \sup _{x \in W} \bar{Q}_{n x}^{\alpha}(C) \leq-\inf _{\alpha \in A_{1}} \inf _{\mu \in C} \bar{I}^{\alpha}(\mu) .
$$

LEMmA 6. If (B1) and (B4) are satisfied, then for each $m>0$,

$$
\bar{C}_{m}=\left\{\mu \in \mathcal{P}(E \times E): \inf _{\alpha \in \mathbb{A}} \bar{I}^{\alpha}(\mu) \leq m\right\}
$$

is a compact subset of $\mathcal{P}(E \times E)$.

To prove an analogue of Proposition 1 the following lemma is used.

Lemma 7. Assume (B1) and (B4) and that for each $\alpha \in \mathbb{A}$ there is a unique invariant measure $\pi_{\alpha}$ of the transition operator $P^{\alpha}(x, d y)$. Then for $\mu \in \mathcal{P}(E \times E)$ the following equivalence is satisfied:

$$
\bar{I}^{\alpha}(\mu)=0 \Leftrightarrow \mu(d x, d y)=P^{\alpha}(x, d y) \pi_{\alpha}(d x) .
$$

To verify this lemma the methods of the proof of Lemma 2.5 of [3] can be used. The details are left to the reader.

Using Lemma 7 we can state an analogue of Proposition 1 combined with Theorem 5 .

THEOREM 6. If (B1), (B3) and (B4) are satisfied, then for any compact sets $W \subset E, A_{1} \subset \mathbb{A}$ and closed set $C \subset \mathcal{P}(E \times E)$ such that $P^{\alpha}(x, d y) \pi_{\alpha}(d x) \notin C$ for $\alpha \in A_{1}$ there are $p>0$ and a positive integer $N$ such that for $n \geq N$,

$$
\sup _{\alpha \in A_{1}} \sup _{x \in W} P_{x}^{\alpha}\left\{n^{-1} \sum_{i=0}^{n-1} \chi_{x_{i}, x_{i+1}}(\cdot) \in C\right\} \leq e^{-n p} .
$$

REMARK 2. If $E$ is compact then the assumptions (B2) and (B4) are clearly satisfied. An analysis of the proofs of Theorems 3 and 6 shows that the constants $p$ can be given in terms of the infima of the rate functions $I^{\alpha}$ and $\bar{I}^{\alpha}$, respectively.

3. Adaptive control with observation of cost. Consider now a controlled Markov process $\left(x_{n}, n \in \mathbb{N}\right)$ with transition operator $P^{\alpha^{0}, v_{n}}\left(x_{n}, \cdot\right)$. The following assumptions are made.

(A1) For $f \in \mathcal{C}(E)$ the mapping

$$
\mathbb{A} \times U \times E \ni(\alpha, v, x) \mapsto P^{\alpha, v} f(x)
$$

is continuous.

(A2) There is a continuous function $\psi: \mathbb{A} \times E \rightarrow \mathbb{R}$ such that $\psi(\alpha, x) \geq 1$ for $\alpha \in \mathbb{A}, x \in E$, the mapping

$$
E \ni x \mapsto \sup _{\alpha \in \mathbb{A}} \sup _{v \in U} \int_{E} \psi(\alpha, y) P^{\alpha, v}(x, d y)
$$


is bounded on compact subsets of $E$ and for each $m>0$ the set

$$
K_{m}:=\left\{x \in E: \inf _{\alpha \in \mathbb{A}} \inf _{v \in U} \frac{\psi(\alpha, x)}{\int_{E} \psi(\alpha, y) P^{\alpha, v}(x, d y)} \leq m\right\}
$$

is compact.

(A3) For $u \in \mathcal{A}_{\mathrm{c}}:=\mathcal{A} \cap C(E, U)$ there is a unique invariant measure $\pi_{\alpha}^{u}$ for $P^{\alpha, u}(x, \cdot)$, and for $c \in \mathcal{C}(E)$ the mapping $\mathbb{A} \ni \alpha \mapsto \pi_{\alpha}^{u}(c)$ is continuous.

(A4) For $\alpha \in \mathbb{A}$ the optimal value of the cost functional $J^{\alpha}$ defined in (1) coincides almost surely with that of

$$
\bar{J}_{c}^{\alpha}\left(\left(v_{n}, n \in \mathbb{N}\right)\right)=\limsup _{n \rightarrow \infty} n^{-1} E_{x}^{\alpha}\left\{\sum_{i=0}^{n-1} c\left(x_{i}, v_{i}\right)\right\} .
$$

Moreover, for any $\varepsilon>0$ there is a finite class $\mathcal{U}(\varepsilon)=\left\{u_{1}, \ldots, u_{r}\right\} \subset$ $\mathcal{A}_{\mathrm{c}}$ of $\varepsilon$-optimal control functions for $\bar{J}^{\alpha}$, that is, for any $\alpha \in \mathbb{A}$ there is a $u_{j} \in \mathcal{U}(\varepsilon)$ such that

$$
\bar{J}_{x}^{\alpha}\left(\left(u_{j}\left(x_{n}\right)\right)\right) \leq \inf _{\left(v_{n}\right)} \bar{J}^{\alpha}\left(\left(v_{n}\right)\right)+\varepsilon .
$$

Furthermore, there is a compact set $W \subset E$ that is positive recurrent for the Markov process $\left(x_{n}, n \in \mathbb{N}\right)$ controlled with any control function of the class $\mathcal{U}(\varepsilon)$.

An analogue of Theorem 3 is given now.

TheOREM 7. Assume that (A1)-(A4) are satisfied and fix $\varepsilon>0$. Then there are $p>0$ and a positive integer $N$ such that for $n \geq N$,

$$
\begin{aligned}
\sup _{k=1, \ldots, r, r} \sup _{\alpha \in \mathbb{A}} \sup _{x \in W} P_{x}^{\alpha u_{k}}\left\{\mid n^{-1} \sum_{j=0}^{n-1} c\left(x_{j}, u_{k}\left(x_{j}\right)\right)\right. & \\
& \left.-\int_{E} c\left(y, u_{k}(y)\right) \pi_{\alpha}^{u_{k}}(d y) \mid \geq \varepsilon\right\} \leq e^{-n p} .
\end{aligned}
$$

Moreover, the following adaptive strategy $\left(\widehat{v}_{j}\right)$ is $\varepsilon$-optimal with probability $\left(1-e^{-n p}\right)^{r}:$ choose $n \geq N$, use the control function $u_{1}$ for $i<T_{1}:=$ $\mathcal{T}(T(0)+n)$, then $u_{2}$ for $T_{1} \leq i<T_{2}:=\mathcal{T}\left(T_{1}+n\right), \ldots$, and $u_{i}$ for $T_{r-1} \leq i<$ $T_{r}:=\mathcal{T}\left(T_{r-1}+n\right)$, where $\mathcal{T}(\tau)$ denotes the first hitting time to the positive recurrent compact set $W$ after the random time $\tau$, determine $\bar{k} \in\{1, \ldots, r\}$ such that

$$
\sum_{j=T_{\bar{k}-1}}^{T_{\bar{k}-1}+n-1} c\left(x_{j}, n_{\bar{k}}\left(x_{j}\right)\right)=\min _{q=1, \ldots, r} \sum_{j=T_{q-1}}^{T_{q-1}+n-1} c\left(x_{j}, u_{q}\left(x_{j}\right)\right)
$$

and after $T_{r}$ use the control function $u_{\bar{k}}$. 
Proof. Note that (38) follows from (24). Then for $x \in W$ and $n \geq N$,

$$
\begin{aligned}
\inf _{\alpha \in \mathbb{A}} P_{x}^{\alpha}\left\{\left|n^{-1} \sum_{j=T_{q-1}}^{T_{q-1}+n-1} c\left(x_{j}, u_{q}\left(x_{j}\right)\right)-\int_{E} c\left(z, u_{q}(z)\right) \pi_{\alpha}^{u_{q}}(d z)\right|\right. & \\
& \leq \varepsilon \text { for } q=1, \ldots, r\} \geq\left(1-e^{-n p}\right)^{r} .
\end{aligned}
$$

Consequently, by (39) for $x \in W$,

$$
\begin{aligned}
\inf _{\alpha \in \mathbb{A}} P_{x}^{\alpha}\left\{\int_{E} c\left(z, u_{\bar{k}}(z)\right) \pi_{\alpha}^{u_{\bar{k}}}(d z) \geq \min _{q=1, \ldots, r} \int_{E} c\left(z, u_{q}(z)\right) \pi_{\alpha}^{u_{q}}(d z)+2 \varepsilon\right\} & \\
& \geq\left(1-e^{-n p}\right)^{r} .
\end{aligned}
$$

Since by $(\mathrm{A} 4), J^{\alpha^{0}}\left(\left(\widehat{v}_{n}\right)\right)=\int_{E} c\left(z, u_{q}(z)\right) \pi_{\alpha}^{u_{\bar{k}}}(d z), P^{\alpha^{0}}$-a.e., it follows that

$$
J^{\alpha^{0}}\left(\left(\widehat{v}_{n}\right)\right) \leq 3 \varepsilon+\inf _{\left(v_{i}\right)} \bar{J}^{\alpha^{0}}\left(\left(v_{i}\right)\right)
$$

with probability $\left(1-e^{-n p}\right)^{r}$.

4. Examples. Two models are described to demonstrate the applicability of the previous results that used large deviations.

Model $I . E$ is a compact metric space. There is a probability measure $\eta$ such that

$$
P^{\alpha, v}(x, B)=\int_{B} p(x, y, \alpha, v) \eta(d y)
$$

for $B \in \mathcal{B}(E)$, the mapping $E \times E \times \mathbb{A} \times U \ni(x, y, \alpha, v) \mapsto p(x, y, \alpha, v)$ is continuous and $p(x, y, \alpha, v)>0$ for $x, y \in E, \alpha \in \mathbb{A}, v \in U$. Clearly (A1)-(A3) are satisfied for this model. Furthermore, there is a finite family of almost optimal controls.

TheOrem 8. For each $\varepsilon>0$ there is a finite class $\mathcal{U}(\varepsilon) \subset \mathcal{A}_{\mathrm{c}}$ of $\varepsilon$-optimal control functions for Model I with cost functionals $\bar{J}^{\alpha}, \alpha \in \mathbb{A}$.

Proof. The proof consists of three steps:

STEP I. Initially it is shown that there is a finite set $\widehat{\mathcal{U}}(\varepsilon)=\left\{u_{1}, \ldots, u_{r}\right\}$ of piecewise constant $\varepsilon$-optimal control functions such that the set of their discontinuity points is of $\eta$-measure zero.

Let $\left\{E_{1}^{n}, E_{2}^{n}, \ldots, E_{d_{n}}^{n}\right\}, n=1,2, \ldots$, be a sequence of partitions of $E$ and $\left\{e_{1}^{n}, e_{2}^{n}, \ldots, e_{d_{n}}^{n}\right\}, n=1,2, \ldots$, be a sequence of their representative elements such that

$$
E=\bigcup_{i=1}^{d_{n}} E_{i}^{n}, \quad E_{i}^{n} \cap E_{j}^{n}=\emptyset \quad \text { for } i \neq j
$$


$\eta\left(\partial E_{i}^{n}\right)=0$, the diameter of $E_{i}^{n}$ is not greater than $1 / n, e_{i}^{n} \in E_{i}^{n}$ for $i=1, \ldots, d,\left\{E_{1}^{n+1}, \ldots, E_{d_{n+1}}^{n+1}\right\}$ is a subpartition of $\left\{E_{1}^{n}, \ldots, E_{d_{n}}^{n}\right\}$ and

$$
\left\{e_{1}^{n}, \ldots, e_{d_{n}}^{n}\right\} \subset\left\{e_{1}^{n+1}, \ldots, e_{d_{n+1}}^{n+1}\right\} .
$$

Moreover, let $p_{n}^{\alpha, v}\left(e_{i}^{n}, e_{j}^{n}\right)=P^{\alpha, v}\left(e_{i}^{n}, e_{j}^{n}\right)$.

Consider now a controlled Markov process on $\left\{e_{1}^{n}, \ldots, e_{d_{n}}^{n}\right\}$ with transition operator $p_{n}^{\alpha, v}\left(e_{i}^{n}, e_{j}^{n}\right)$. By Lemma 3 of [1] for given $\varepsilon>0$ there is a finite set $\left\{\bar{u}_{1}^{n}, \ldots, \bar{u}_{r_{n}}^{n}\right\}$ of control functions such that

$$
\begin{array}{r}
\sup _{\alpha \in \mathbb{A}} \sup _{1 \leq i \leq r_{n}} \sup _{1 \leq j \leq d_{n}}\left[\bar{w}_{\alpha}^{n}\left(e_{j}^{n}\right)-\lambda_{\alpha}^{n}-\sum_{i=1}^{d_{n}} \bar{w}_{\alpha}^{n}\left(e_{l}^{n}\right) p_{n}^{\alpha, \bar{u}_{i}^{n}\left(e_{j}^{n}\right)}\left(e_{j}^{n}, e_{l}^{n}\right)\right. \\
\left.+c\left(e_{j}^{n}, \bar{u}_{i}^{n}\left(e_{j}^{n}\right)\right)\right] \leq \frac{\varepsilon}{2}
\end{array}
$$

where $\lambda_{\alpha}^{n}$ and $\bar{w}_{\alpha}^{n}$ are the optimal value and the Bellman function of the corresponding cost functional $\bar{J}^{\alpha}$, respectively.

Let $u_{j}^{n}(x)=\bar{u}_{j}^{n}\left(e_{l}^{n}\right), w_{\alpha}^{n}(x)=\bar{w}_{\alpha}^{n}\left(e_{l}^{n}\right)$, and $P_{n}^{\alpha, v}(x, \cdot)=P^{\alpha, v}\left(e_{l}^{n}, \cdot\right)$ for $x \in E_{l}^{n}, l=1, \ldots, d_{n}$. It follows that

$$
\sup _{\alpha \in \mathbb{A}} \min _{1 \leq j \leq r_{n}} \sup _{x \in E}\left\{w_{\alpha}^{n}(x)-\lambda_{\alpha}^{n}-P_{n}^{\alpha, u_{i}^{n}(x)} w_{\alpha}^{n}(x)+c_{n}\left(x, u_{i}^{n}(x)\right)\right\} \leq \varepsilon / 2
$$

with $c_{n}(x, v)=c\left(e_{l}^{n}, v\right)$ for $x \in E_{l}^{n}$ and $v \in U$. Note, moreover, that $\lambda_{\alpha}^{n}$ is also the optimal value of the cost functional $\bar{J}^{\alpha}$ corresponding to the controlled Markov process on $E$ with transition operator $P_{n}^{\alpha, v}(x, \cdot)$.

By Lemma 3.3.3 of [6] for $u \in \mathcal{A}, n=1,2, \ldots$, there exist probability measures $\pi_{\alpha}^{u}$ and $\pi_{\alpha}^{u, n}$ such that for $x \in E$ and $B \in \mathcal{B}(E)$,

$$
\left|\left(P^{u, \alpha}\right)^{k}(x, B)-\pi_{\alpha}^{u}(B)\right| \leq(1-d)^{k-1}
$$

and

$$
\left|\left(P_{n}^{u, \alpha}\right)^{k}(x, B)-\pi_{\alpha}^{u, n}(B)\right| \leq(1-d)^{k-1}
$$

with

$$
d=\inf _{x, y \in E} \inf _{\alpha \in \mathbb{A}} \inf _{v \in U} p(x, y, \alpha, v) .
$$

An analysis of the proof of Proposition 1 of [9] shows that there exists a constant $K>0$ that is independent of $u \in \mathcal{A}, \alpha \in \mathbb{A}$ and $n \in \mathbb{N}$, for which

$$
\left\|\pi_{\alpha}^{u}-\pi_{\alpha}^{u, n}\right\|_{\mathrm{var}} \leq \sup _{x \in E} K\left\|P_{n}^{u, \alpha}(x, \cdot)-P^{u, \alpha}(x, \cdot)\right\|_{\mathrm{var}}
$$

where $\|\cdot\|_{\text {var }}$ denotes the variation norm.

Since by the continuity of the transition density $p(x, y, \alpha, v)$,

$$
\lim _{n \rightarrow \infty} \sup _{u \in \mathcal{A}} \sup _{\alpha \in \mathbb{A}} \sup _{x \in E} K\left\|P_{n}^{u, \alpha}(x, \cdot)-P^{u, \alpha}(x, \cdot)\right\|_{\text {var }}=0,
$$


for $\lambda_{\alpha}=\inf _{u \in \mathcal{A}} \int_{E} c(x, u(x)) \pi_{\alpha}^{u}(d x)$ it follows that

$$
\sup _{\alpha \in \mathbb{A}}\left|\lambda_{\alpha}-\lambda_{\alpha}^{n}\right| \leq \sup _{\alpha \in \mathbb{A}}\left\|\pi_{\alpha}^{u}-\pi_{\alpha}^{u, n}\right\|_{\mathrm{var}}+\sup _{x \in E, v \in U}\left|c(x, v)-c_{n}(x, v)\right| \rightarrow 0
$$

as $n \rightarrow \infty$. Therefore, for sufficiently large $n$ from (42), (44), and (45) we obtain

$$
\sup _{\alpha \in \mathbb{A}} \min _{1 \leq j \leq r_{n}} \sup _{x \in E}\left\{w_{\alpha}^{n}(x)-\lambda_{\alpha}-P^{\alpha, u_{i}^{n}(x)} w_{\alpha}^{n}(x)+c_{n}\left(x, u_{i}^{n}(x)\right)\right\} \leq \varepsilon,
$$

which means (cf. the proof of Theorem 3.2 .2 of [6]) that $\widehat{\mathcal{U}}(\varepsilon)=\left\{u_{1}^{n}, \ldots\right.$ $\left.\ldots, u_{r}^{n}\right\}$ is a set of $\varepsilon$-optimal control functions for the original Markov process. It follows from the construction that the set of discontinuity points of the above control functions is of $\eta$-measure 0 .

STEP II. It is clear that for $i=1, \ldots, r$ there is a sequence $u_{i}(n) \in \mathcal{A}_{\mathrm{c}}$, $n=1,2, \ldots$, such that

$$
\lim _{n \rightarrow \infty} \eta\left(\left\{z \in E: u_{i}(z) \neq u_{i}(n)(z)\right\}\right)=0 .
$$

We claim that for any bounded Borel function $f: E \rightarrow \mathbb{R}$ such that the set of discontinuity points of $f$ is of $\eta$-measure 0 , and for $i=1, \ldots, r$,

$$
\lim _{n \rightarrow \infty} \sup _{\alpha \in \mathbb{A}}\left|\pi_{\alpha}^{u_{i}}(f)-\pi_{\alpha}^{u_{i}(n)}(f)\right|=0 .
$$

Assume that contrary to (48) there is a sequence $\left(\alpha_{n}, n \in \mathbb{N}\right)$ from $\mathbb{A}$ such that $\alpha_{n} \rightarrow \alpha \in \mathbb{A}$ and for some $i \in\{1, \ldots, r\}$,

$$
\left|\pi_{\alpha_{n}}^{u_{i}}(f)-\pi_{\alpha_{n}}^{u_{i}(n)}(f)\right|>\delta>0
$$

for sufficiently large $n$.

By the tightness of a suitably chosen subsequence $n_{k}$ it follows that

$$
\pi_{\alpha_{n_{k}}}^{u_{i}\left(n_{k}\right)} \Longrightarrow \pi
$$

where $\pi$ is a probability measure on $E$. We now show that $\pi$ is invariant for the transition operator $P^{a, u_{i}}(x, \cdot)$. In fact, for $g \in \mathcal{C}(E)$ it follows that

$$
\begin{aligned}
\left|\pi(g)-\pi\left(P^{\alpha, u_{i}} g\right)\right| \leq & \left|\pi(g)-\pi_{\alpha_{n_{k}}}^{u_{i}\left(n_{k}\right)}(g)\right| \\
& +\left|\pi_{\alpha_{n_{k}}}^{u_{i}\left(n_{k}\right)}(g)-\pi_{\alpha_{n_{k}}}^{u_{i}\left(n_{k}\right)}\left(P^{\alpha_{n_{k}}, u_{i}\left(n_{k}\right)} g\right)\right| \\
& +\left|\pi_{\alpha_{n_{k}}}^{u_{i}\left(n_{k}\right)}\left(P^{\alpha_{n_{k}}, u_{i}\left(n_{k}\right)} g\right)-\pi_{\alpha_{n_{k}}}^{u_{i}\left(n_{k}\right)}\left(P^{\alpha, u_{i}\left(n_{k}\right)} g\right)\right| \\
& +\left|\pi_{\alpha_{n_{k}}}^{u_{i}\left(n_{k}\right)}\left(P^{\alpha, u_{i}\left(n_{k}\right)} g\right)-\pi_{\alpha_{n_{k}}}^{u_{i}\left(n_{k}\right)}\left(P^{\alpha, u_{i}} g\right)\right| \\
& +\left|\pi_{\alpha_{n_{k}}}^{u_{i}\left(n_{k}\right)}\left(P^{\alpha, u_{i}} g\right)-\pi\left(P^{\alpha, u_{i}} g\right)\right| \\
= & I_{1 k}+I_{2 k}+I_{3 k}+I_{4 k}+I_{5 k} .
\end{aligned}
$$

Clearly $I_{1 k} \rightarrow 0$ as $k \rightarrow \infty$, and $I_{2 k}=0$. Moreover,

$$
I_{3 k} \leq|g| \sup _{x, y \in E} \sup _{v \in U}\left|p\left(x, y, \alpha_{n_{k}}, v\right)-p(x, y, \alpha, v)\right| \rightarrow 0
$$


as $k \rightarrow \infty$. Define

$$
M=\sup _{x, y \in E} \sup _{\alpha \in \mathbb{A}} \sup _{v \in U} p(x, y, \alpha, v) .
$$

Since $\pi_{\alpha_{n_{k}}}^{u_{i}\left(n_{k}\right)}$ is an invariant measure it follows that $\pi_{\alpha_{n_{k}}}^{u_{i}\left(n_{k}\right)}(\cdot) \leq M \eta(\cdot)$. Therefore, $\pi(\cdot) \leq M \eta(\cdot)$. Consequently, by (47), $I_{4 k} \rightarrow 0$ as $k \rightarrow \infty$, and since the set of discontinuity points of $P^{\alpha, u_{i}} g$ is of $\eta$-measure $0, I_{5 k} \rightarrow 0$ as $k \rightarrow \infty$.

By the uniqueness of the invariant measure it follows that $\pi=\pi_{\alpha}^{u_{i}}$. Since $\pi$ does not depend on a particular subsequence it follows that

$$
\pi_{\alpha_{n}}^{u_{i}(n)} \Longrightarrow \pi_{\alpha}^{u_{i}}
$$

as $n \rightarrow \infty$. Using similar arguments it also follows that

$$
\pi_{\alpha_{n}}^{u_{i}} \Longrightarrow \pi_{\alpha}^{u_{i}}
$$

as $n \rightarrow \infty$, which together with (51) contradicts (49). Thus, (48) is satisfied.

STEP III. The following inequality is elementary:

$$
\begin{aligned}
\sup _{\alpha \in \mathbb{A}}\left|\int_{E} c\left(x, u_{i}(x)\right) \pi_{\alpha}^{u_{i}}(d x)-\int_{E} c\left(x, u_{i}(n)(x)\right) \pi_{\alpha}^{u_{i}(n)}(d x)\right| \\
\leq \sup _{\alpha \in \mathbb{A}}\left[\left|\int_{E} c\left(x, u_{i}(x)\right) \pi_{\alpha}^{u_{i}}(d x)-\int_{E} c\left(x, u_{i}(x)\right) \pi_{\alpha}^{u_{i}(n)}(d x)\right|\right. \\
\left.+\left|\int_{E}\left(c\left(x, u_{i}(n)(x)\right)-c\left(x, u_{i}(x)\right)\right) \pi_{\alpha}^{u_{i}(n)}(d x)\right|\right] \\
=I_{1 n}+I_{2 n} .
\end{aligned}
$$

By (48) clearly $I_{1 n} \rightarrow 0$ as $n \rightarrow \infty$. Since by (47) also

$$
I_{2 n} \leq\|c\| M \eta\left(\left\{x \in E: u_{i}(n)(x) \neq u_{i}(x)\right\}\right) \rightarrow 0
$$

as $n \rightarrow \infty$ with $M$ defined in (50), for sufficiently large $n$, for $\alpha \in \mathbb{A}$ and $i=1, \ldots, r$ it follows that

$$
\bar{J}_{x}^{\alpha}\left(u_{i}(n)\right) \leq \bar{J}_{x}^{\alpha}\left(u_{i}\right)+\varepsilon \leq \lambda_{\alpha}+2 \varepsilon .
$$

Thus, the set $\left\{u_{1}(n), \ldots, u_{r}(n)\right\}$ contains $2 \varepsilon$-optimal control functions for the cost functional $\bar{J}^{\alpha}$ with $\alpha \in \mathbb{A}$.

Therefore, (A4) is also satisfied.

Model II. Let $E=\mathbb{R}^{d}$. Assume $\left(x_{n}, n \in \mathbb{N}\right)$ satisfies the following recursive formula:

$$
x_{n+1}=f\left(x_{n}, \alpha^{0}, v_{n}\right)+g\left(x_{n}\right) w_{n}
$$

where $f: \mathbb{R}^{d} \times \mathbb{A} \times U \rightarrow \mathbb{R}^{d}$ and $g: \mathbb{R}^{d} \rightarrow \mathbb{R}^{d} \times \mathbb{R}^{d}$ are continuous bounded functions, there is a continuous bounded inverse matrix $g^{-1}$, and $w_{n}$ is a sequence of independent, identically distributed standard Gaussian random 
variables. By the continuity of $f, g$, and $g^{-1}$ it follows that (A1) and (A3) (cf. [9]) are satisfied. In (A2) let $\psi(\alpha, x)=x^{2}+1$ so that

$$
\frac{\psi(\alpha, x)}{\int_{E} \psi(\alpha, y) P^{\alpha v}(x, d y)}=\frac{x^{2}+1}{f^{2}(x, \alpha, v)+g^{2}(x)}
$$

and $K_{m}$ is compact if

$$
\sup _{\alpha \in \mathbb{A}} \sup _{v \in U}\left[f^{2}(x, \alpha, v)+g^{2}(x)\right]=o\left(x^{2}\right)
$$

as $x^{2} \rightarrow \infty$, and, in particular, if $f$ and $g$ are bounded.

Hence (A2) is satisfied. Just as for Model I there is the following result.

ThEOREM 9. For given $\varepsilon>0$ there is a finite family $\mathcal{U}(\varepsilon)$ of $\varepsilon$-optimal control functions for Model II with cost functionals $\bar{J}^{\alpha}, \alpha \in \mathbb{A}$.

P r o o f. By Lemma 1 of [9] there are a finite measure $\eta$ that is absolutely continuous with respect to Lebesgue measure in $\mathbb{R}^{d}$ and a constant $M$ such that for $x \in \mathbb{R}^{d}, \alpha \in \mathbb{A}$ and $v \in U$,

$$
\eta(\cdot) \leq P^{\alpha, v}(x, \cdot) \leq M \eta(\cdot) .
$$

Consider now a sequence of partitions $\left\{E_{1}^{n}, \ldots, E_{d_{n}}^{n}\right\}, n=1,2, \ldots$, of $\mathbb{R}^{d}$ and representative elements $\left\{e_{1}^{n}, \ldots, e_{d_{n}}^{n}\right\}, n=1,2, \ldots$, such that

$$
\mathbb{R}^{d}=\bigcup_{i=1}^{d_{n}} E_{i}^{n}, \quad E_{i}^{n} \cap E_{j}^{n}=\emptyset \quad \text { for } i \neq j,
$$

$\eta\left(\partial E_{i}^{n}\right)=0, e_{i}^{n} \in E_{i}^{n}$ for $i=1, \ldots, d_{n}$, the diameter of $E_{i}^{n}$ is not greater than $1 / n$ for $i=1, \ldots, d_{n}-1, e_{d_{n}}^{n} \subset\left\{x \in \mathbb{R}^{d}:\|x\|>n\right\},\left\{E_{1}^{n+1}, \ldots, E_{d_{n+1}}^{n+1}\right\}$ is a subpartition of $\left\{E_{1}^{n}, \ldots, E_{d_{n}}^{n}\right\}$ and

$$
\left\{e_{1}^{n}, \ldots, e_{d_{n}}^{n}\right\} \subset\left\{e_{1}^{n+1}, \ldots, e_{d_{n+1}}^{n+1}\right\} .
$$

Now the methods of the proof of Theorem 7 can be used. Note only that the value of $d$ in (43) is now replaced by $\eta\left(\mathbb{R}^{d}\right)$.

Thus, (A1)-(A4) are satisfied. Moreover, for any $u \in \mathcal{A}$ and transition operator $P^{\alpha u(x)}(x, \cdot)$, the assumption (B4) is satisfied with the function $\bar{\psi}(\alpha, x, y)=x^{2}+y^{4}+1$.

5. Adaptive control with estimation. Consider first the case of Model I. For $u \in \mathcal{A}_{\mathrm{c}}$ and $\alpha, \alpha^{\prime} \in \mathbb{A}$ let

$$
K^{u}\left(\alpha, \alpha^{\prime}\right):=\int_{E} \int_{E} p(x, y, \alpha, u(x)) \log p\left(x, y, \alpha^{\prime}, u(x)\right) \mu(d y) \pi_{u}^{\alpha}(d x) .
$$

Fix $\varepsilon>0$. By the proofs of Propositions 7 and 4 in [5] there is $\delta>0$ such that for $\alpha, \alpha^{\prime} \in \mathbb{A}$ and $u \in \mathcal{U}(\varepsilon)$, if $K^{u}(\alpha, \alpha)-K^{u}\left(\alpha, \alpha^{\prime}\right) \leq \delta$ then 


$$
\left\|\pi_{\alpha}^{u}-\pi_{\alpha^{\prime}}^{u}\right\|_{\mathrm{var}} \leq \varepsilon
$$

By the continuity of $K^{u}\left(\alpha, \alpha^{\prime}\right)$ and the compactness of $\mathbb{A}$ there are a finite sequence $\alpha_{1}, \ldots, \alpha_{k}$ and $\eta>0$ such that for $\alpha \in \mathbb{A}$ there is $\alpha_{j}$ such that $\alpha \in B_{\eta}\left(\alpha_{j}\right)=\left\{\bar{\alpha} \in \mathbb{A}: \varrho_{A}\left(\bar{\alpha}, \alpha_{j}\right) \leq \eta\right\}$ and for $u \in \mathcal{U}(\varepsilon)$

$$
\begin{aligned}
K^{u}(\alpha, \alpha)-K^{u}\left(\alpha, \alpha_{j}\right) & \leq \delta / 2, \\
\sup _{\bar{\alpha} \in \mathbb{A}}\left|K^{u}\left(\alpha_{j}, \bar{\alpha}\right)-K^{u}(\alpha, \bar{\alpha})\right| & \leq \delta / 16 .
\end{aligned}
$$

For $u \in \mathcal{U}(\varepsilon)$ let

$$
\begin{aligned}
\widehat{\alpha}_{n}^{u}=\left\{\alpha_{j}: \prod_{i=0}^{n-1} p\left(x_{i}, x_{i+1}, u\left(x_{i}\right), \alpha_{j}\right)\right. & \\
& =\max _{q=1, \ldots, k} \prod_{i=1}^{n-1} p\left(x_{i}, x_{i+1}, u\left(x_{i}\right), \alpha_{q}\right) \\
& \left.>\max _{q \leq j-1} \prod_{i=0}^{n-1} p\left(x_{i}, x_{i+1}, u\left(x_{i}\right), \alpha_{q}\right)\right\} .
\end{aligned}
$$

THEOREM 10. For Model I there exist a $p>0$ and a positive integer $n \geq N$ such that for $u \in \mathcal{U}(\varepsilon)$,

$$
\inf _{\alpha \in \mathbb{A}} \inf _{x \in E} P_{x}^{\alpha}\left\{\left\|\pi_{\alpha}^{u}-\pi_{\widehat{\alpha}_{n}^{u}}\right\|_{\mathrm{var}} \leq \varepsilon\right\} \geq 1-k e^{-n p},
$$

where $\widehat{\alpha}_{n}^{u}$ is given by (57).

Proof. Let

$$
\begin{aligned}
C^{u}\left(\alpha_{i}, \alpha_{j}\right)= & \{l \in \mathcal{P}(E \times E): \\
& \left.\left|\int_{E \times E} \log p\left(x, y, u(x), \alpha_{j}\right) l(d x, d y)-K^{u}\left(\alpha_{i}, \alpha_{j}\right)\right| \geq \delta / 8\right\} .
\end{aligned}
$$

If $\alpha \in B_{\eta}\left(\alpha_{i}\right)$ then $P^{\alpha u}(x, d y) \pi_{\alpha}^{u}(d y) \notin C^{u}\left(\alpha_{i}, \alpha_{j}\right)$ because

$$
\int_{E \times E} \log p\left(x, y, u(x), \alpha_{j}\right) P^{\alpha u}(x, d y) \pi_{\alpha}^{u}(d x)=K^{u}\left(\alpha, \alpha_{j}\right)
$$

and by $(57),\left|K^{u}\left(\alpha, \alpha_{j}\right)-K\left(\alpha_{i}, \alpha_{j}\right)\right| \leq \delta / 16$. Therefore, by Theorem 6 there are a $p>0$ and a positive integer $N$ such that for $n \geq N, i, j=1, \ldots, k$ and $u \in \mathcal{U}(\varepsilon)$,

$$
\begin{aligned}
\sup _{\alpha \in B_{\eta}\left(\alpha_{i}\right)} \sup _{x \in E} P_{x}^{\alpha u}\left\{\mid n^{-1} \sum_{m=0} \log p\left(x_{m},\right.\right. & \left.x_{m+1}, u\left(x_{m}\right), \alpha_{j}\right) \\
- & \left.K^{u}\left(\alpha_{i}, \alpha_{j}\right) \mid \geq \delta / 8\right\} \leq e^{-n p} .
\end{aligned}
$$


Then from (57) for $n \geq N$ and $i, j=1, \ldots, k$,

$$
\begin{array}{r}
\sup _{\alpha \in B_{\eta}\left(\alpha_{i}\right)} \sup _{x \in E} P_{x}^{\alpha u}\left\{\mid n^{-1} \sum_{m=0} \log p\left(x_{m}, x_{m+1}, u\left(x_{m}\right), \alpha_{j}\right)\right. \\
\left.-K^{u}\left(\alpha, \alpha_{j}\right) \mid \geq \delta / 4\right\} \leq e^{-n p} .
\end{array}
$$

Since

$$
n^{-1} \sum_{m=0}^{n-1} \log p\left(x_{m}, x_{m+1}, u\left(x_{m}\right), \widehat{\alpha}_{n}^{u}\right) \geq n^{-1} \sum_{m=0}^{n-1} \log p\left(x_{m}, x_{m+1}, u\left(x_{m}\right), \alpha_{j}\right)
$$

for $j=1, \ldots, k$, using (60) it follows that

$$
K^{u}\left(\alpha, \widehat{\alpha}_{n}^{u}\right)+\delta / 4 \geq K^{u}\left(\alpha, \alpha_{j}\right)-\delta / 4
$$

for $j=1, \ldots, k$ with probability $1-k e^{-n p}$, for $\alpha \in \mathbb{A}$ and $u \in \mathcal{U}(\varepsilon)$.

Consequently, using (56) for $u \in \mathcal{U}(\varepsilon)$ we get

$$
\inf _{\alpha \in \mathbb{A}} \inf _{x \in E} P_{x}^{\alpha u}\left\{K^{u}(\alpha, \alpha)-K^{u}\left(\alpha, \widehat{\alpha}_{n}^{u}\right) \leq \delta\right\} \geq 1-k e^{p n}
$$

and by (55) it follows that (58) is satisfied.

Now consider the following adaptive strategy: Choose $n \geq N$ where $N$ is as in Theorem 10 and test each control function of the family $\mathcal{U}(\varepsilon)$ for $n$ units of time. Then for $q=1, \ldots, r$ determine

$$
\begin{aligned}
\bar{\alpha}_{q}=\left\{\alpha_{j}: \sum_{i=n(q-1)}^{n q-1} p\left(x_{i}, x_{i+1}, u_{q}\left(x_{i}\right), \alpha_{j}\right)\right. & \\
& =\max _{j^{\prime}=1, \ldots, k} \sum_{i=n(q-1)}^{n q-1} p\left(x_{i}, x_{i+1}, u_{q}\left(x_{i}\right), \alpha_{j^{\prime}}\right) \\
& \left.>\max _{j^{\prime} \leq j-1} \sum_{i=n(q-1)}^{n q-1} p\left(x_{i}, x_{i+1}, u_{q}\left(x_{i}\right), \alpha_{j^{\prime}}\right)\right\}
\end{aligned}
$$

and find $p \in\{1, \ldots, r\}$ such that

$$
\int_{E} c\left(y, u_{p}(y)\right) \pi_{\bar{\alpha}_{p}}^{u_{p}}(d y)=\min _{q=1, \ldots, r} \int_{E} c\left(y, u_{p}(y)\right) \pi_{\bar{\alpha}_{p}}^{u_{p}}(d y) .
$$

By Theorem 10 the following corollary easily follows:

Corollary 1. The control strategy $\widehat{v}_{j}=u_{p}\left(x_{j}\right)$ for $j \geq n r$ is $2 \varepsilon\|c\|-$ optimal with probability $\left(1-k e^{-n p}\right)^{r}$.

For Model II define, for $u \in \mathcal{A}_{\mathrm{c}}$,

$$
K^{u}\left(\alpha, \alpha^{\prime}\right)=\iint_{\mathbb{R}^{d} \mathbb{R}^{d}}\left\|\left(y-f\left(x, \alpha^{\prime}, u(x)\right)\right) g^{-1}(x)\right\|^{2} P^{\alpha u(x)}(x, d y) \pi_{\alpha}^{u}(d x)
$$


and

$$
\begin{aligned}
& K_{m}^{u}\left(\alpha, \alpha^{\prime}\right) \\
& =\int_{\mathbb{R}^{d}} \int_{\mathbb{R}^{d}}\left(\left\|\left(y-f\left(x, \alpha^{\prime}, u(x)\right)\right) g^{-1}(x)\right\|^{2} \wedge m\right) P^{\alpha u(x)}(x, d y) \pi_{\alpha}^{u}(d x) .
\end{aligned}
$$

By Proposition 1 of [9], $K^{u}\left(\alpha, \alpha^{\prime}\right)$ and $K_{m}^{u}\left(\alpha, \alpha^{\prime}\right)$ are continuous functions in their two variables. Moreover, the following continuity property is satisfied.

Lemma 8. If $u \in \mathcal{A}_{\mathrm{c}}$ then

$$
\sup _{\alpha, \alpha^{\prime} \in \mathbb{A}}\left|K^{u}\left(\alpha, \alpha^{\prime}\right)-K_{m}^{u}\left(\alpha, \alpha^{\prime}\right)\right| \rightarrow 0
$$

as $m \rightarrow \infty$.

Proof. Suppose that for $\alpha_{m} \rightarrow \alpha$ and $\alpha_{m}^{\prime} \rightarrow \alpha^{\prime}$ there is $\delta>0$ such that

$$
\left|K^{u}\left(\alpha_{m}, \alpha_{m}^{\prime}\right)-K_{m}^{u}\left(\alpha_{m}, \alpha_{m}^{\prime}\right)\right|>\delta .
$$

Since

$$
K^{u}\left(\alpha, \alpha^{\prime}\right)=\int_{\mathbb{R}^{d}} \mathbb{E}\left\{\left\|\left(f(x, \alpha, u(x))-f\left(x, \alpha^{\prime}, u(x)\right)\right) g^{-1}(x)+\xi\right\|^{2}\right\} \pi_{\alpha}^{u}(d x)
$$

and

$$
\begin{aligned}
K_{m}^{u}\left(\alpha, \alpha^{\prime}\right)=\int_{\mathbb{R}^{d}} \mathbb{E}\{\min \{ & \|(f(x, \alpha, u(x)) \\
& \left.\left.\left.-f\left(x, \alpha^{\prime}, u(x)\right)\right) g^{-1}(x)+\xi \|^{2}, m\right\}\right\} \pi_{\alpha}^{u}(d x),
\end{aligned}
$$

where $\xi$ is an $N(0,1)$ random variable and $\mathbb{E}$ is expectation, and by Proposition 1 of [9], $\left\|\pi_{\alpha_{m}}^{u}-\pi_{\alpha}^{u}\right\|_{\text {var }} \rightarrow 0$, it follows that $K_{m}^{u}\left(\alpha_{m}, \alpha_{m}^{\prime}\right) \rightarrow K^{u}\left(\alpha, \alpha^{\prime}\right)$ and $K^{u}\left(\alpha_{m}, \alpha_{m}^{\prime}\right) \rightarrow K^{u}\left(\alpha, \alpha^{\prime}\right)$ as $m \rightarrow \infty$. This is a contradiction.

Lemma 9. For $\varepsilon>0$ and $u \in \mathcal{A}_{\mathrm{c}}$ there is a $\delta>0$ such that if $K^{u}\left(\alpha, \alpha^{\prime}\right)$ $\leq \delta$ then $\left\|\pi_{\alpha}^{u}-\pi_{\alpha^{\prime}}^{u}\right\|_{\mathrm{var}} \leq \varepsilon$.

Proof. If the lemma does not hold then $K^{u}\left(\alpha_{n}, \alpha_{n}^{\prime}\right) \rightarrow 0, \alpha_{n} \rightarrow \alpha$, $\alpha_{n}^{\prime} \rightarrow \alpha^{\prime}$ and $\left\|\pi_{\alpha_{n}}^{u}-\pi_{\alpha_{n}^{\prime}}^{u}\right\|_{\text {var }} \geq \varepsilon$ for $n=1,2, \ldots$ and an $\varepsilon>0$. From Lemma 8 it follows that $K\left(\alpha, \alpha^{\prime}\right)=0$. By (65) and the proof of Theorem 9 it follows that $f(x, \alpha, u(x))=f\left(x, \alpha^{\prime}, u(x)\right)$ for almost all $x \in \mathbb{R}^{d}$ with respect to $d$-dimensional Lebesgue measure. Since $f$ and $u$ are continuous functions, $f(x, \alpha, u(x))=f\left(x, \alpha^{\prime}, u(x)\right)$ for all $x \in \mathbb{R}^{d}$, and consequently $\pi_{\alpha}^{u}=\pi_{\alpha^{\prime}}^{u}$, a contradiction.

Combining Lemmas 9 and 8 gives the following corollary.

Corollary 2. For $\varepsilon>0$ there are $m>0$ and $\delta>0$ such that for $u \in \mathcal{U}(\varepsilon)$, if $K_{m}^{u}\left(\alpha, \alpha^{\prime}\right)<\delta$ then $\left\|\pi_{\alpha}^{u}-\pi_{\alpha^{\prime}}^{u}\right\|_{\text {var }} \leq \varepsilon$. 
Fix $\varepsilon>0$ and take $m$ as in Corollary 2. There are a finite set $\alpha_{1}, \ldots, \alpha_{k} \in$ $\mathbb{A}$ and $\eta>0$ such that for $\alpha \in B_{\eta}\left(v_{j}\right)$ and $u \in \mathcal{U}(\varepsilon)$,

$$
K_{m}^{u}\left(\alpha, \alpha_{j}\right) \leq \delta / 2
$$

and

$$
\sup _{\bar{\alpha} \in \mathbb{A}}\left|K_{m}^{u}\left(\alpha_{j}, \bar{\alpha}\right)-K_{m}^{u}(\alpha, \bar{\alpha})\right| \leq \delta / 16
$$

Let

$$
\begin{aligned}
\widehat{\alpha}_{m}^{u}=\left\{\alpha_{j}: \sum_{i=0}^{n-1}\right. & \left\|\left(x_{i+1}-f\left(x_{i}, \alpha_{j}, u\left(x_{i}\right)\right)\right) g^{-1}\left(x_{i}\right)\right\|^{2} \\
& =\min _{q=1, \ldots, k} \sum_{i=1}^{n-1}\left\|\left(x_{i+1}-f\left(x_{i}, \alpha_{q}, u\left(x_{i}\right)\right)\right) g^{-1}\left(x_{i}\right)\right\|^{2} \\
& \left.\leq \min _{q \leq j-1} \sum_{i=0}^{n-1}\left\|\left(x_{i+1}-f\left(x_{i}, \alpha_{q}, u\left(x_{i}\right)\right)\right) g^{-1}\left(x_{i}\right)\right\|^{2}\right\} .
\end{aligned}
$$

Using Theorem 6, Corollary 2 and (67)-(69) as in the case of Model I yields the following theorem.

TheOREM 11. Given Model II for a given compact set $W \subset \mathbb{R}^{d}$ there are $p>0$ and a positive integer $N$ such that for $n \geq N$ and $u \in \mathcal{U}(\varepsilon)$,

$$
\inf _{\alpha \in \mathbb{A}} \inf _{\alpha \in W} P_{x}^{\alpha}\left\{\left\|\pi_{\alpha}^{u}-\pi_{\widehat{\alpha}_{n}^{u}}^{u}\right\|_{\text {var }} \leq \varepsilon\right\} \geq 1-k e^{-p n} .
$$

Let $W \subset \mathbb{R}^{d}$ be a compact set that is positive recurrent for each $u \in \mathcal{U}(\varepsilon)$. Choose $n \geq N$ and use control $u_{1}$ for $i<T_{1}, u_{2}$ for $T_{1} \leq i<T_{2}, \ldots$, and $u_{r}$ for $T_{r-1} \leq i<T_{r}$ where $T_{i}$ is defined as in Theorem 7 .

For $q=1, \ldots, r$ let

$$
\begin{aligned}
\bar{\alpha}_{q}=\left\{\alpha_{j}:\right. & \sum_{i=T_{q-1}}^{T_{q-1}+n}\left\|\left(x_{i+1}-f\left(x_{i}, \alpha_{j}, u\left(x_{i}\right)\right)\right) g^{-1}\left(x_{i}\right)\right\|^{2} \\
& =\min _{j^{\prime}=1, \ldots, n} \sum_{i=T_{q-1}}^{T_{q-1}+n}\left\|\left(x_{i+1}-f\left(x_{i}, \alpha_{j^{\prime}}, u\left(x_{i}\right)\right)\right) g^{-1}\left(x_{i}\right)\right\|^{2} \\
& \left.<\min _{j^{\prime} \leq j-1} \sum_{i=T_{q-1}}^{T_{q-1}+n}\left\|\left(x_{i+1}-f\left(x_{i}, \alpha_{j^{\prime}}, u\left(x_{i}\right)\right)\right) g^{-1}\left(x_{i}\right)\right\|^{2}\right\} .
\end{aligned}
$$

Find $p \in\{1, \ldots, r\}$ such that

$$
\int_{E} c\left(u, u_{p}(y)\right) \pi_{\bar{\alpha}_{p}}^{u_{p}}(d y)=\min _{q=1,2, \ldots, r} \int_{E} c\left(y, u_{q}(y)\right) \pi_{\bar{\alpha}_{p}}^{u_{p}}(d y) .
$$

Just as for Model I, the following corollary is obtained. 
COROllaRY 3 . The control $\widehat{v}_{j}=u_{p}\left(x_{j}\right)$ for $j \geq T_{r}$ is $2 \varepsilon\|c\|$-optimal with probability $\left(1-k e^{-n p}\right)^{r}$.

\section{References}

[1] G. B. Di Masi and L. Stettner, Bayesian ergodic adaptive control of discrete time Markov processes, Stochastics Stochastics Rep. 54 (1995), 301-316.

[2] M. D. Donsker and S. R. S. Varadhan, Asymptotic evaluation of certain Markov process expectations for large time, I, Comm. Pure Appl. Math. 28 (1975), 1-47.

[3] -, 一, Asymptotic evaluation of certain Markov process expectations for large timeIII, ibid. 29 (1976), 389-461.

[4] M. Duflo, Formule de Chernoff pour des chaînes de Markov (d'après Donsker et Varadhan), in: Grandes déviations et applications statistiques, Séminaire Orsay 1977-78, Astérisque 68 (1979), 99-124.

[5] T. E. Duncan, B. Pasik-Duncan and L. Stettner, Discretized maximum likelihood and almost optimal control of ergodic Markov models, SIAM J. Control Optim. 36 (1998), 422-446.

[6] O. Hernández-Lerma, Adaptive Markov Control Processes, Springer, 1976.

[7] N. Maigret, Majorations de Chernoff pour des chaînes de Markov contrôlées, Z. Wahrsch. Verw. Gebiete 51 (1980), 133-151.

[8] - Statistiques des chaînes controlés Felleriennes, in: Grandes déviations et applications statistiques, Séminaire Orsay, 1977-1978, Astérisque 68 (1979), 143-169.

[9] /L. Stettner, On nearly self-optimizing strategies for a discrete-time uniformly ergodic adaptive model, Appl. Math. Optim. 27 (1993), 161-177.

T. E. Duncan, B. Pasik-Duncan

Department of Mathematics

University of Kansas

Lawrence, KS 66045, U.S.A.

E-mail: duncan@math.ukans.edu bozenna@kuhub.cc.ukans.edu
Łukasz Stettner Department of Mathematics University of Kansas Lawrence, KS 66045, U.S.A. E-mail: stettner@impan.gov.pl 